\title{
Skull Base Reconstruction with Titanium Mesh for Benign Complex Anterior Skull Base Tumors: Case Series and Review of the Literature
}

\author{
Taha MS* and Alnemari HH
}

Department of Neurosurgery, King Fahad Specialist Hospital-Dammam, P.O. Box: 15215 MBC 35, Amer Bin Thabet St., Dammam 31444, Kingdom of Saudi Arabia

\begin{abstract}
Aim: The aim of the study was to evaluate the role of titanium mesh used in combination with other synthetic materials in providing a rigid support for reconstruction of anterior skull base defect and preventing CSF leak.

Material and methods: All Cases of complex benign anterior skull base lesions operated between December 2008 and December 2013 (5 years) which had titanium mesh for reconstruction were reviewed. Six operations were performed on five patients for large anterior skull base meningioma ( 3 cases) and fibrous dysplasia ( 2 cases). The main complaint was exophthalamous in 4 patients, and one presented with nasal blockage due to ethmoidal and nasal invasion. The reconstructions were performed by using titanium mesh with pericranial flaps, synthetic dural grafts, and surgical glue. Routine lumbar drain was not used. Three patients had previous surgeries. Three patients were female, and the age range was 4 to 55 . We had a minimum of 12 months follow up. The incidence of CSF leak, infections, and other surgical morbidity related to the reconstruction were recorded. The status of exophthalamous post operatively was also observed.

Results: We had no incidence of CSF leak or infection. One case of recurrent psuedomeningocele was encountered and it required repeated aspiration, then lumbar drain, and finally insertion of subgalial-peritoneal shunt. Exophthalamous improved in 3 patients and remain the same in one.
\end{abstract}

Conclusion: Use of titanium mesh with local flaps or synthetic dural graft and surgical glue is safe, simple, and effective for reconstruction of anterior skull base defects caused by benign tumor or fibrous dysplasia.

Keywords: Skull base; Reconstruction; Titanium mesh; Meningiomas; Fibrous Dysplasia; Neurofibromatosis

\section{Introduction}

Reconstruction of anterior cranial base defects is often necessary following resection of tumors, destructive infectious lesions, fibrous dysplasia, and severe trauma. The aim of reconstruction is to provide a watertight barrier between intra-cranial contents, para-nasal sinuses, and orbital cavities. Following reconstruction, no CSF leak, or herniation of the brain tissue into the para-nasal sinuses or the orbits should be encountered [1].

Various methods of repairing the floor of the anterior cranial fossa have been described. Some reports have indicated that reconstruction using soft tissue alone is adequate in most patients [2]. Pericranial and galeal flaps are most commonly used as local soft tissue barriers. Free pieces of fat, muscle, and fascia have been also used in patients who do not have sufficient local soft tissue [3]. Synthetic materials such as dural grafts or surgical glue are currently used to provide a watertight sealant; it can be used alone or with others [4].

To provide more rigid support, some authors have also reported using of bone graft or metallic mesh in conjunction with soft tissue repair. The rigid grafts may be advantageous in preventing the development of brain herniation into the sinuses or the orbits in patients with large skull base defects and/or longer expected survival [5].

Free vascularized (microvascular) flaps are usually used following resection of malignant skull base lesions; where much larger defects are expected in the bone and the soft tissue after resection [6]. Some authors however, are advocating the use of these flaps in non-malignant cases especially after recurrence of CSF leak following repair by local flap [7].

\section{Materials and Method}

The data was collected retrospectively for patients with benign lesions who underwent skull base surgery and reconstruction by using titanium mesh.

Data includes demographics, pre and post-op imaging, histopathology reports, techniques details including skull base repair and reconstruction, and post-op complication with their management. The cosmetic results were assessed by patient satisfaction and clinical evaluation.

5 cases were studied (female/ male: $3 / 2$ ), age range from 4-55, pathologies were: 2 cases of Intraosseous meningiomas, 2 cases with fibrous dysplasia associated with Neurofibromatosis type1, and one case of invasive olfactory groove meningioma. Patient's characteristics are detailed in Table 1.

\section{Case Illustration and Surgical Technique}

\section{Case 1}

This 54-year-old female presented with 4 years of progressive left exophthalamous and feeling of hard lump at the side of the head. No visual impairment. Imaging revealed diffuse bone lesion involving the left temporal bone (squamous part), sphenoid wing and left orbit

*Corresponding author: Mahmoud Sadek Taha, Consultant Neurosurgeon, Department of Neurosurgery, King Fahad Specialist Hospital-Dammam, P.O. Box 15215 MBC 35, Amer Bin Thabet St., Dammam 31444, Kingdom of Saudi Arabia Tel: 00966 -542757185; E-mail: mtaha66@hotmail.com

Received January 12, 2016; Accepted January 28, 2016; Published February 05, 2016

Citation: Taha MS, Alnemari HH (2016) Skull Base Reconstruction with Titanium Mesh for Benign Complex Anterior Skull Base Tumors: Case Series and Review of the Literature. J Brain Tumors Neurooncol 1: 104. doi: 10.4172/2475-3203.1000104

Copyright: $\odot 2016$ Taha MS, et al. This is an open-access article distributed under the terms of the Creative Commons Attribution License, which permits unrestricted use, distribution, and reproduction in any medium, provided the original author and source are credited. 


\begin{tabular}{|c|c|c|c|c|c|c|c|}
\hline Case no. & Age/sex & Diagnosis & location & $\begin{array}{l}\text { previous } \\
\text { surgery }\end{array}$ & main complain & surgical approach & follow up \\
\hline Case 1 & $54 / F$ & Intraosseous meningioma & left sphenoid/orbit & no & exophthalamous & Bi-frontal craniotomy flap & 3 years \\
\hline Case 1 & $54 / F$ & $\begin{array}{c}\text { Recurrent Intraosseous } \\
\text { meningioma }\end{array}$ & left sphenoid/orbit & yes & exophthalamous & Bi-frontal craniotomy flap & 1 year \\
\hline Case 2 & $49 / F$ & Intraosseous meningioma & right sphenoid/ orbit & no & exophthalamous & Bi-frontal craniotomy flap & 4.5 years \\
\hline Case 3 & $48 / F$ & Invasive meningioma & $\begin{array}{l}\text { olfactory groove/ethmoid } \\
\text { and nasal cavity }\end{array}$ & yes & nasal blockage & $\begin{array}{l}\text { Bi-frontal craniotomy flap and } \\
\text { trans-nasal endoscopy }\end{array}$ & 3 years \\
\hline Case 4 & $16 / \mathrm{M}$ & Fibrous dysplasia In NF1 & right sphenoid/ orbit & yes & $\begin{array}{l}\text { facial deformity/ } \\
\text { exophthalamous }\end{array}$ & Bi-frontal craniotomy flap & 4 years \\
\hline Case 5 & $4 / \mathrm{M}$ & Fibrous dysplasia In NF1 & left sphenoid/ orbit & no & $\begin{array}{l}\text { facial deformity/ } \\
\text { exophthalamous }\end{array}$ & Bi-frontal craniotomy flap & 2 years \\
\hline
\end{tabular}

Table 1: Patient's demographics, pathology, location, surgical approach, and follow up.

consisting with primary extra-dural intraosseous meningioma (Figure 1). The patients underwent bi-frontal flap and extra-dural partial resection of the lesion. The main bulk of the temporal and the orbital parts of the tumor were removed with a high speed drill, and the bony defects were replaced by titanium mesh which was previously fashioned to fit the size and the shape of the defect. The mesh was secured with mini-plate and screws (Figure 2). Dural tear was encountered and this was repaired with local pericranial flap and surgical glue. Histopathology confirmed intraosseous meningioma. Radiotherapy was offered to the patient after surgery, but she declined. 3 years later she came with recurrent symptoms (mainly exophthalaomous), and imaging showed recurrence of her tumor. Same technique was used again for her recurrent tumor. The patient had a large dural tear during surgery, and it was repaired by using artificial dura and surgical glue. A week after surgery she developed CSF wound, and it was treated with aspiration and head bandage. The collection recurred after 2 weeks. This time, we tried a lumbar drain for a week. Again recur after several weeks, and at this point we decided to insert a subgalial- peritoneal shunt (valveless type). The problem resolved after the shunt. Pathology showed the same histology. Radiotherapy was offered again to the patient but she is still refusing the treatment.

\section{Case 2}

This is a 49 -year-old female with 2 years history of progressive right exophthalamous. She had no visual decline and no other complains. Imaging of her head revealed a diffuse bony lesion involving the right sphenoid and orbital roof. The patient had almost identical appearance of first case (except it was in the other side) and she was operated by using the same approach. Also, same techniques were used to repair the skull defect. Histology confirmed intraosseous meningioma similar the first case. She had a good recovery with no complication during the 4.5 years follow up.

\section{Case 3}

This is a 48-year-old female with previous history of olfactory groove meningioma operated 4 years back. She presented with nasal blockage and difficulty in breathing. Imaging of her brain revealed a small intra-cranial recurrence at the previous site with a large extracranial invasion into the ethmoid sinus and the nasal cavity (Figure 3).

The case was treated surgically by a joint approach; trans-cranially via bi-frontal flap and trans-nasally via endoscopic approach (by our ENT team). The lesion was totally excised. The dura was breached and repaired with artificial dura and glue. The defect at the skull base was covered by 4 layers; mucosal flap from the nose, pieces of fat, and surgical glue for re-enforcement. Then a piece of titanium mesh was fashioned and used to cover the bony defect (Figure 4). Interestingly, the pathology of the tumor reported meningiothelial meningioma
WHO grade 1. No CSF leak or other complications were encountered during the 3 years follow up.

\section{Case 4}

This is a 15-year-old male who is known to have neurofibromatosis type 1 . He presented to us with a major deformity involving the right side of his face. He had a large facial plexiform neurofibroma affecting the skin and causing excessive skin redundancy with severe right exophthalamous. Surprisingly, he had intact vision and partial eye movement. Imaging of his head showed a large developmental defect in the right sphenoid wing region. Nearly, most of the right sphenoid wing and the roof of the orbit were lacking and this has caused a large brain herniation into the orbit and severe exophthalamous. No intracranial tumor was seen on imaging (Figure 5). The patient had one surgical attempt in the past and the results were not satisfactory.

Our surgery was planned and discussed with the plastic team. The first step of surgery was to build a new right orbit to accommodate the globe. A large right fronto-temporal flap was done. The large bony defect was exposed, and we found the herniated dura with its frontal lobe content in the orbital cavity. The dura was stuck to the displaced orbital contents. We decided here to open the dura and retract the brain backward to allow a space for titanium mesh which will be replacing the missing skull base.

A large local pericranial flap was then used with a dural graft to separate the brain from the new titanium mesh. Surgical glue was also used to allow watertight closure of the dura defect. The mesh was fashioned in a pyramidal shape to replace the missing orbit, and it was secured by using mini-plates and screws to the remaining of the orbital roof and the floor of the middle fossa (Figure 6). At a later stage, the patient was operated by the plastic surgeons for his facial tumor and skin redundancy. No post-operative complication was observed during the 4 years follow up.

Intravenous antibiotics are always given at induction of anesthesia and re-given if surgery last more than 4 hours. Dexamethasone is also given as a routine shortly before surgery and continues for a week post operatively. No lumbar drain was used in any of the cases during the reconstructive surgery.

\section{Case 5}

This was a 4-year-old male child with known diagnosis of NF1. He presented to us with 2 years history of left exophthalmos with a bony bulge at the left temporal area. The child has also a primary glaucoma and increase in the size of his left eye. Imaging revealed a defect in the roof of orbit with brain herniation (Figure $7 \mathrm{~A}, \mathrm{~B}$ ). We operated in a similar fashion to case no. 4. A left temporo-frontal flap was made and the defect was identified. The herniated dura with its brain content 
Citation: Taha MS, Alnemari HH (2016) Skull Base Reconstruction with Titanium Mesh for Benign Complex Anterior Skull Base Tumors: Case Series and Review of the Literature. J Brain Tumors Neurooncol 1: 104. doi: 10.4172/2475-3203.1000104
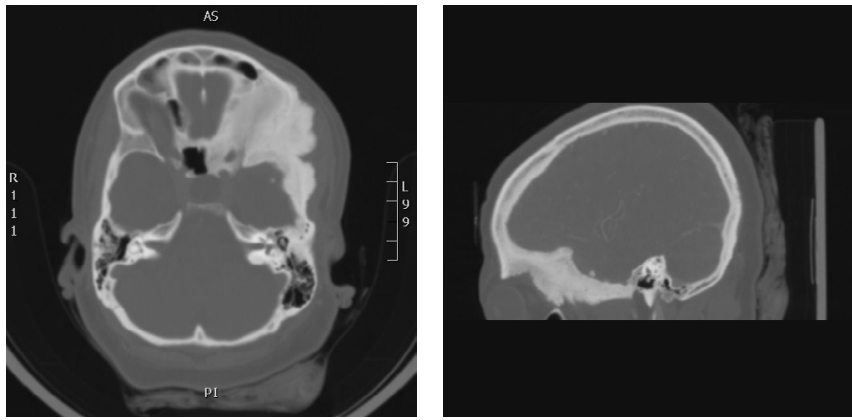

Figure 1: A and B: CT scans of case no. 1 showing sphenoid wing skull base primary extra-dural intraosseous meningioma with orbital involvement.

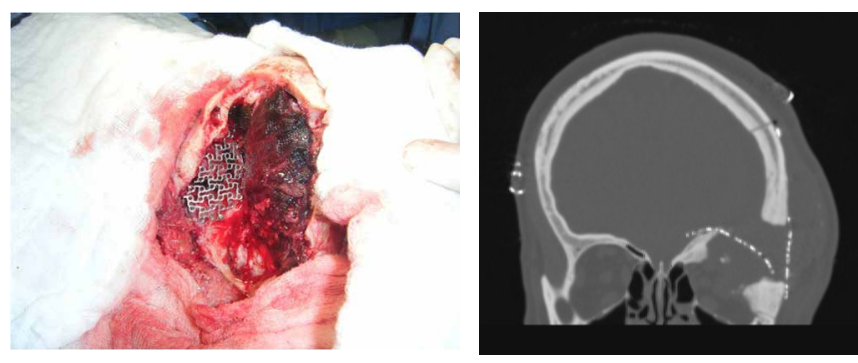

Figure 2: A: Intra-operative picture for the same case showing the orbital defect with the titanium mesh used. B: Post-operative CT for case no. 1.

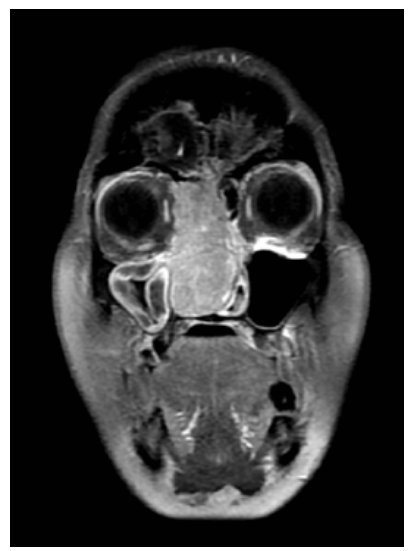

Figure 3: MRI of case no. 3 showing the lesion in anterior skull base with a large invasion to the ethmoid and nasal cavity. The skull base defect is also seen in the left cribriform plate.

was pulled out of the orbital cavity and the defect was covered with a titanium mesh (Figure 8). The child reported no complication during the 2 years follow up and the exophthalmos remains stable.

Intravenous antibiotics are always given at induction of anesthesia and re-given if surgery last more than 4 hours. Dexamethasone is also given as a routine shortly before surgery and continues for a week post operatively. No lumbar drain was used in any of the cases during the reconstructive surgery.

\section{Results}

All cases were operated between December 2008 and December
2013. The minimum follow up we have was 12 months (range from 1 to 5 years). No adverse reaction related to the use of titanium mesh was observed. None of the patients developed CSF leak or meningitis or other intra-cranial infection during follow up. We had only one case of recurrent CSF wound collection (pseudomeningocele) in the case number 1 as mentioned previously.

Patients with exophthalamous showed good results after surgery except case number 6 which is the pediatric case of NF1 due to the

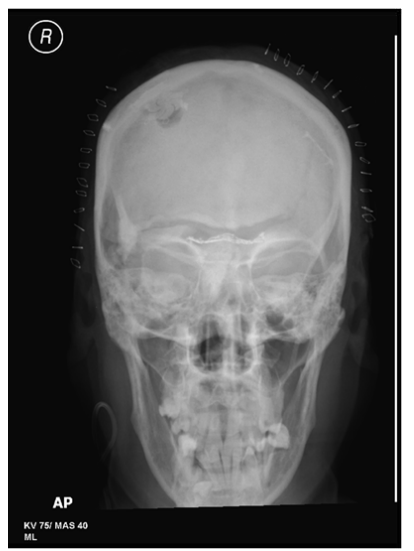

Figure 4: Plain x-ray for case no. 3 showing the titanium mesh covering the cribriform plate defect. The mesh was fixed bilaterally to the roof of the orbit.
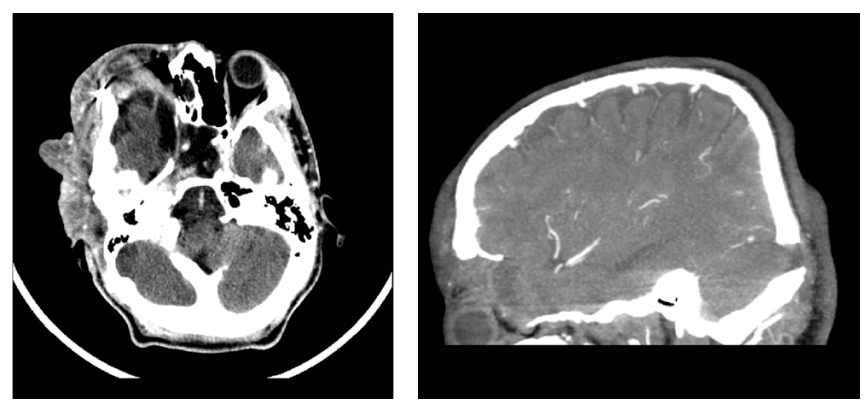

Figure 5: A and B: CT brain of case no. 4 showing the large bony defect in the right sphenoid wing and the roof of the orbit with a large brain herniation into the orbit. This herniation was the case for his exophthalamous. The facial plexiform neurofibroma is also seen. No intracranial tumor was seen on imaging. A small defect was seen in the occipital bone from a previous surgery.
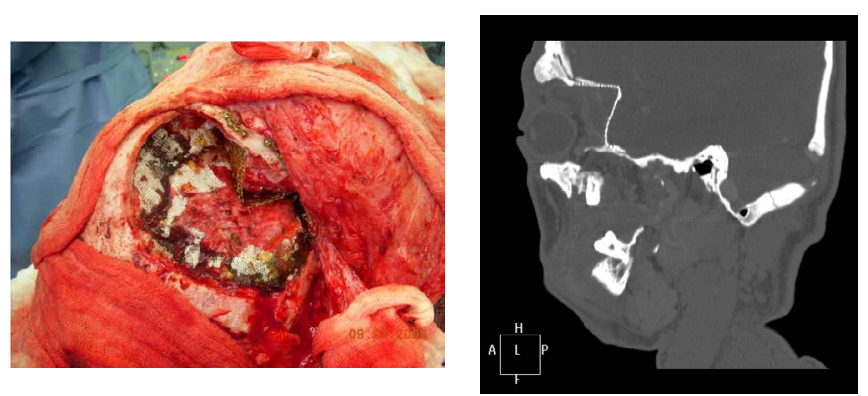

Figure 6: A: Intra-operative image of case no. 4 showing the titanium mesh replacing the missing right orbit and separating the orbital content from the dura. B: Post-op CT scan of case no. 4 showing the final position of the mesh. 
Citation: Taha MS, Alnemari HH (2016) Skull Base Reconstruction with Titanium Mesh for Benign Complex Anterior Skull Base Tumors: Case Series and Review of the Literature. J Brain Tumors Neurooncol 1: 104. doi: 10.4172/2475-3203.1000104

Page 4 of 5
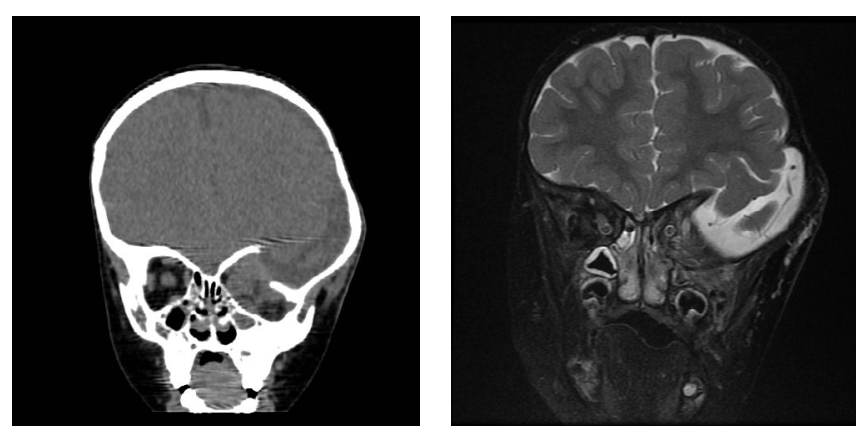

Figure 7: A: CT brain of case no. 5 showing the bony defect in the left orbital roof with brain herniation and the bony deformity in the left temporal bone. B: MRI of case n. 5 showing the same finding as the CT.

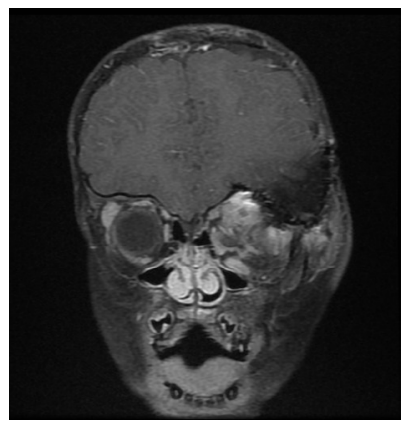

Figure 8: MRI of case no. 5 after surgery showing the improvement of the brain herniation and the artifacts caused by the titanium mesh.

presence of primary glaucoma of the eye and increase in the size of the globe.

\section{Discussion}

The integrity of the skull base could be inflicted by several benign pathologies. The most common seen are: meningioma, invasive pituitary adenoma, craniopharyngioms, and fibrous dysplasia. Surgical resection of these lesions doesn't usually cause large defects in the skull base, and in most cases simple repair by local flaps or tissue is enough $[1,3]$.

Primary extradural meningiomas constitute 1 to $2 \%$ of all meningiomas. Primary intraosseous meningioma (cases 1 and 2) is a term used to describe a subset of extradural meningiomas that arise in bone. This type of meningioma represents approximately two thirds of all extradural meningiomas. Skull base intraoseous meningiomas are usually slow growing and painless. They usually give symptoms related to mass effects to the cranial nerves or the orbit [8]. Wide surgical excision is the treatment of choice. Ideally, cranial reconstruction should be performed together with the resection. Total resection of this skull base lesion may not be possible, in which case decompression of vital neural structures is performed. Radiotherapy is recommended for residual and symptomatic tumor [9].

Intra-dural meningioma with extra-cranial invasion (secondary extra-dural meningioma) is not unusual (case 3) and can be seen in up to $20 \%$ of benign meningiomas (WHO grade 1). Orbit, outer table and skin, para-nasal sinuses and nasal cavity, and para-pharyngeal region are among the common sites for extra-cranial extension [10].

Sphenoid wing dysplasia (case 4 and 5) is a condition that is pathognomonic to neurofibromatosis type 1 , and it occurs in $4-11 \%$ of all patients. In some reported cases of fibrous dysplasia the brain can herniate into the orbital cavity, and causes pulsatile proptosis or exophthalamous $[11,12]$.

The use of titanium mesh provides a simple method for reconstruction of large anterior skull base defects. It provides a rigid support which can prevent brain herniation in patients with expected long survival. It should be used according to some reports after establishing a water tight closure of the dura [5]. A second layer below the mesh is important when para-nasal sinuses are breached to separate the mesh from para-nasal sinuses. This will theoretically reduce the risk of infection [13]. This second layer is not necessary in cases of orbitosphenoidal reconstruction $[12,14]$.

Biron, reported a novel technique for large anterior skull base defects with compromised recipient bed from repeated infection, multiple surgeries, or previous radiation. The technique combined the use of titanium mesh with radial forearm free flap sandwich [7].

All previous studies of the use titanium mesh for anterior skull base reconstruction have reported a favorable outcome as shown in Table 2 [5,7-16].

\section{Conclusion}

The use of Titanium mesh can provide a solid barrier and separation between the cranium and the orbit or the nasal cavity. It should however be used with local flaps or artificial dura with surgical glue to achieve water tight closure of the dura. We found it safe and effective in preventing CSF leak and infection.

\begin{tabular}{|c|c|c|c|}
\hline Study & Pathologies & F/ up & no. of patients \\
\hline Badie (5) & Trauma, benign, malignant & 22 months & 13 \\
\hline Sinah (13) & All pathologies & long (not defined) & 20 \\
\hline Lotfy (15) & Fibrous dysplasia & 1 year & no complications \\
\hline Snyder (16) & Fibrous dysplasia & $6-15$ months & no complications \\
\hline Luetjens (14) & sphenoorbital meningiomas & long (not defined) & no complications \\
\hline Biron (7) & rauma with recurrent meningitis & $1-4.5$ months & 3 \\
\hline Current study & Fibrous dysplasia, meningioma & 1 year & 3 \\
\hline
\end{tabular}

Table 2: Literature review of series describing benign skull base lesions with titanium mesh reconstruction. 
Citation: Taha MS, Alnemari HH (2016) Skull Base Reconstruction with Titanium Mesh for Benign Complex Anterior Skull Base Tumors: Case Series and Review of the Literature. J Brain Tumors Neurooncol 1: 104. doi: 10.4172/2475-3203.1000104

Page 5 of 5

\section{Reference}

1. Gil Z, Avraham A, Leonor LT, Avi K, Nevo M, et al. (2007) A comprehensive algorithm for anterior skull base reconstruction after oncological resections. Skull Base 17: 25-37.

2. McCutcheon LE, Blacklock JB, Weber RS, DeMonte F, Moser RP, et al. (1996) Anterior transcranial resection of tumors of the paranasal sinuses: surgical techniques and results. Neurosurgery $38: 471-480$

3. Nibu K, Sasaki T, Kawahara N (1998) complications of craniofacial surgery for tumors involving the anterior cranial base. Neurosurgery 42: 455-462.

4. Kerr JT, Chu FW, Bayles SW (2005) Cerebrospinal fluid rhinorrhea: diagnosis and management. Otolaryngol clin north am 38: 597-611.

5. Badie B, Preston JK, Hartig G (2000) Use of titanium mesh for reconstruction of large anterior cranial base defects. J Neurosurg 93: 711-714.

6. Pusic AL, Chen CM, Patel S (2007) Microvascular reconstruction of the skul base: a clinical approach to surgical defects classification and flap selection. Skull Base 17: 5-15.

7. Biron VL, Gross M, Broad R, Seikaly H, Wright ED (2012) Radial forearm free flap with titanium mesh sandwich reconstruction in complex anterior skull base defects. J Craniofac Surg 23: 1763-1765.

8. Elader JB, Atkinson R, Zee C, Chen TC (2007) Primary intraosseous meningioma. Neurosurgery Focus 23 E13: 1-9.
9. Mattox ABS, Hughes B, Oleson J, Readon D, McLendon R, et al. (2011) Treatment recommendation for primary extradural meningioma. Cancer 1: 2438.

10. Perry A, Louis DN, Scheithaner BW, Budka H, Von Deimlin A (2007) WHO classification of tumors of the nervous system. Lyon IARC pp 164-172.

11. Jacquemin C, Bosley TM, Svedberg H (2003) Orbit deformities in craniofacia neurofibromatosis type 1. Am J Neuroradiol 24: 1678-1682.

12. Dale EL, Strait TA, Sargent LA (2014) Orbital reconstruction for pulsatile exophthalmos secondary to sphenoid wing dysplasia. Ann Plast Surg 72 Suppl 2: 107-111.

13. Sinha UK, Johnson TE, Crockett D, Vadapali S, Grenen P (2002) Three-layer reconstruction for large defects of the anterior skull base. Laryngoscope 112 424-427.

14. Luetjens, G, Krauss JK, Brandis A, Nakamura M (2011) Bilateral sphenoorbita hyperostotic meningiomas with proptosis and visual impairment: a therapeutic challenge. Report of three patients and review of the literature. Clin Neuro Neurosurg 113: 859-863.

15. Lotfy M, Xu R, McGirt M, Saker S, Ayoub B, et al. (2010) Reconstruction of skull base defects in sphenoid wing dysplasia associated with neurofibromatosis I with titanium mesh. Clin Neurol Neurosurg 112: p. 909-914.

16. Snyder BJ, Hanieh A, Trott JA, David DJ (1998) Transcranial correction of orbital orbital neurofibromatosis. Plast Reconstr Surg 102: 633-642. 